\title{
ON THE FAILURE OF THE URYSOHN-MENGER SUM FORMULA FOR COHOMOLOGICAL DIMENSION
}

\author{
A. N. DRANIŠNIKOV, D. REPOVŠ, AND E. V. ŠČEPIN
}

(Communicated by James E. West)

\begin{abstract}
We prove that the classical Urysohn-Menger sum formula, $\operatorname{dim}(A \cup B) \leq \operatorname{dim} A+\operatorname{dim} B+1$, which is also known to be true for cohomological dimension over the integers (and some other abelian groups), does not hold for cohomological dimension over an arbitrary abelian group of coefficients. In particular, we prove that there exist subsets $A, B \subset \mathbb{R}^{4}$ such that $4=\operatorname{dim}_{\mathbb{Q} / \mathbb{Z}}(A \cup B)>\operatorname{dim}_{\mathbb{Q} / \mathbb{Z}} A+\operatorname{dim}_{\mathbb{Q} / \mathbb{Z}} B+1=3$.
\end{abstract}

\section{INTRODUCTION}

One of the key properties of covering dimension is that the Urysohn-Menger sum formula holds: Let $A, B \subset X$ be arbitrary subsets of a separable metric space $X$. Then

$$
\operatorname{dim}(A \cup B) \leq \operatorname{dim} A+\operatorname{dim} B+1 .
$$

It was shown by Rubin [13] that this is also true for cohomological dimension with integer coefficients, $G=\mathbb{Z}$

$$
\operatorname{dim}_{G}(A \cup B) \leq \operatorname{dim}_{G} A+\operatorname{dim}_{G} B+1 .
$$

Recently, Dydak and Walsh [9] have proved the formula (2) for the case when $G$ is either the integers modulo a prime $p, \mathbb{Z}_{p}$, or the integers localized at a subset $l$ of primes, $\mathbb{Z}_{l}$, provided that $\operatorname{dim}_{G} A \geq 2$ and $\operatorname{dim}_{G} B \geq 2$.

The purpose of the present paper is to exhibit an example which shows that formula (2) does not generalize to cohomological dimension over arbitrary abelian groups $G$. Theorem 1.1 also solves a long-standing problem in cohomological dimension theory (cf. [11, Problem 10]).

Theorem 1.1. There exist subsets $A, B \subset \mathbb{R}^{4}$ such that:

(i) $\operatorname{dim}_{\mathbb{Q} / \mathbb{Z}} A=1$;

Received by the editors July 20, 1992.

1991 Mathematics Subject Classification. Primary 55M10, 54F45; Secondary 54D35, 54G20.

Key words and phrases. Urysohn-Menger sum formula, cohomological dimension, Boltyanskii compacta, Bockstein inequalities, dimension type, compactification, completion.

The second author was supported in part by a grant from the Ministry of Science and Technology of the Republic of Slovenia. 
(ii) $\operatorname{dim}_{\mathbb{Q} / \mathbb{Z}} B=1$; and

(iii) $\mathbb{R}^{4}=A \cup B$.

Remark 1.1. Theorem 1.1 remains valid if one replaces the group $\mathbb{Q} / \mathbb{Z}$ with the group $\mathbb{Z}_{p^{\infty}}, p$ any prime, where $\mathbb{Z}_{p^{\infty}}$ is the $p$-adic circle, $\mathbb{Z}_{p^{\infty}}=\mathbb{Q} / \mathbb{Z}_{(p)}$, and $\mathbb{Z}_{(p)}$ is the $p$-localization of the integers, $\mathbb{Z}_{(p)}=\left\{\frac{m}{n} \mid n\right.$ is not divisible by p\} (cf. [6]).

Our example from Theorem 1.1 shows that the equation

$$
\operatorname{dim}_{G}(X \cup Y)-\operatorname{dim}_{G} X-\operatorname{dim}_{G} Y=k
$$

has a solution for $G=\mathbb{Q} / \mathbb{Z}$ and $k=2$. On the other hand, note that Dydak [8] has shown that $k \leq 3$, provided that $\operatorname{dim}_{G} X \geq 2$ and $\operatorname{dim}_{G} Y \geq 2$.

\section{Preliminaries}

A subset $Y$ of a space $S$ is said to be negligible with respect to some compactum $X$ ( $X$-negligible) (cf. [6]), if mappings of $X$ into $S$ are approximable by mappings whose images miss $Y$. Compacta $X$ and $Y$ are said to be of the same dimension type, DIM $X=\operatorname{dim} Y$, if $\operatorname{dim}_{G} X=\operatorname{dim}_{G} Y$, for every abelian group $G$. Note that this is equivalent to the requirement that $\operatorname{dim}(X \times Z)=\operatorname{dim}(Y \times Z)$, for every compactum $Z$ (cf. [6]).

Proposition 2.1. Let $X \subset \mathbb{R}^{n}$ be any compactum. Then there exists a subset $X^{\sigma} \subset \mathbb{R}^{n}$ such that :

(i) $X^{\sigma}$ is $\sigma$-compact;

(ii) $\operatorname{DIM} X^{\sigma}=\operatorname{DIM} X$; and

(iii) for every compactum $Y \subset \mathbb{R}^{n} \backslash X^{\sigma}, \operatorname{dim}(X \times Y)<n$.

Proof. Consider a sequence $\left\{\tau_{k}\right\}_{k \in \mathbb{N}}$ of triangulations of $\mathbb{R}^{n}$ such that $\lim _{k \rightarrow \infty}$ mesh $\tau_{k}=0$, and let $I=\left\{l: \mathbb{R}^{n} \rightarrow \mathbb{R}^{n} \mid l\right.$ is simplicial with respect to some subdivision of $\tau_{k}$ and has compact support $\}$. Set $X^{\sigma}=L(X)$, where $L(X)=\bigcup\{l(X) \mid l \in L\}$. Clearly, $X^{\sigma}$ is $\sigma$-compact, so assertion (i) is verified. Next, since $l$ is a PL map, it follows by [6] that, for every compactum $C \subset \mathbb{R}^{n}$, $\operatorname{DIM} l(C)=\operatorname{DIM} C$. Therefore, $\operatorname{DIM} X^{\sigma}=\operatorname{DIM} X$, by the Countable Sum Theorem, so (ii) also holds.

It remains to verify (iii). Let $Y \subset \mathbb{R}^{n} \backslash X^{\sigma}$ be any compactum. Then $Y$ is $X$-negligible in $\mathbb{R}^{n}$, so by [4], $\operatorname{dim}(X \times Y)<n$.

We conjecture that the inequality $\operatorname{DIM}(Y) \leq \operatorname{DIM}\left(\mathbb{R}^{n} \backslash X^{\delta}\right)$ holds for every compactum $Y$ such that $\operatorname{dim}(X \times Y)<n$ (cf. [6, Theorem 1.14]).

Proposition 2.2. Let $X^{\sigma} \subset \mathbb{R}^{n}$ be a $\sigma$-compact subset of $\mathbb{R}^{n}$ such that, for every prime $p, \operatorname{dim}_{\mathbb{Z}_{p}} X^{\sigma}=1$. Then there exists $a G_{\delta}$-set $X^{\delta} \subset \mathbb{R}^{n}$ such that $X^{\sigma} \subset X^{\delta}$ and $\operatorname{dim}_{\mathbb{Q} / \mathbb{Z}} X^{\delta}=\operatorname{dim}_{\mathbb{Z}_{p}} X^{\delta}=1$, for all primes $p$.

For the proof of Proposition 2.2 we shall require two lemmas.

Lemma 2.1. Suppose that $X$ is a $\sigma$-compact metric space such that, for every prime $p, \operatorname{dim}_{\mathbb{Z}_{p}} X=1$. Then there exists a metrizable compactification $c X$ of $X$ such that $\operatorname{dim}_{\mathbb{Z}_{p}} c X=1$, for all primes $p$.

Proof. This is a result of Švedov (cf. [11, Chapter VII]).

Lemma 2.2. Let $S$ and $T$ be complete metric spaces and $h: A \rightarrow B$ any homeomorphism between subsets $A \subset S$ and $B \subset T$. Then there exist $G_{\delta}$-sets $\tilde{A} \subset S$ 
and $\widetilde{B} \subset T$ such that $A \subset \widetilde{A}, B \subset \widetilde{B}$, and $h$ extends to a homeomorphism $\tilde{h}: \widetilde{A} \subset \widetilde{B}$.

Proof. This is a classical result of Lavrent'ev [12] (cf. [10, p. 335]).

Proof of Proposition 2.2. By Lemma 2.1, there is a metrizable compactification $c X^{\sigma}$ of $X^{\sigma}$ such that $\operatorname{dim}_{\mathbb{Z}_{p}} c X^{\sigma}=1$, for all primes $p$. Hence, by the Bockstein inequalities [1] (cf. [11,6]), $\operatorname{dim}_{\mathbb{Q} / \mathbb{Z}} c X^{\sigma}=1$. Consider the canonical inclusion $i: X^{\sigma} \hookrightarrow c X^{\sigma}$.

Apply Lemma 2.2 to get $G_{\delta}$-sets $\tilde{A} \subset \mathbb{R}^{n}$ and $\widetilde{B} \subset c X^{\sigma}$, such that $X^{\sigma} \subset \tilde{A}$ and $i\left(X^{\sigma}\right) \subset \widetilde{B}$, and an extension of $i$ to a homeomorphism $\tilde{i}: \widetilde{A} \rightarrow \widetilde{B}$. Then $X^{\delta}=\tilde{A}$ is the required $G_{\delta}$-set from the assertion. Indeed, since $\operatorname{dim}_{\mathbb{Q} / \mathbb{Z}} c X^{\sigma}=$ 1 , we have that $\operatorname{dim}_{\mathbb{Q} / \mathbb{Z}} \widetilde{B}=1$, so $\operatorname{dim}_{\mathbb{Q} / \mathbb{Z}} X^{\delta}=1$.

\section{Proof of Theorem 1.1}

By [2], there exists a compactum $X$ such that $\operatorname{dim}_{\mathbb{Z}_{(p)}} X=\operatorname{dim} X=2$ and $\operatorname{dim}_{\mathbb{Z}_{p}} X=\operatorname{dim}_{\mathbb{Q}} X=1$, for all primes $p$. Therefore, by [11], $\operatorname{dim}(X \times X)=3$, so it follows by [5] (cf. also [14]) that $X$ embeds in $\mathbb{R}^{4}$. Also, by the Bockstein inequalities [1] (cf. [11,6]), $\operatorname{dim}_{\mathbb{Z}_{p \infty}} X=1$, for all primes $p$, so $\operatorname{dim}_{\mathbb{Q} / \mathbb{Z}} X=1$, by the Bockstein theorem [11].

Let $X^{\sigma}$ be as in Proposition 2.1. Let $A=X^{\delta}$, where $X^{\delta}$ is the completion of $X^{\sigma}$, guaranteed by Proposition 2.2, with $\operatorname{dim}_{\mathbb{Z}_{p}} X^{\delta}=1$, for all primes $p$, and $\operatorname{dim}_{\mathbb{Q} / \mathbb{Z}} X^{\delta}=1$. Define $B=\mathbb{R}^{4} \backslash X^{\delta}$. Thus $B$ is of $F_{\sigma}$-type. In order to complete the proof it suffices, by the Countable Sum Theorem [11], to verify the following.

Assertion. For every compactum $C \subset B, \operatorname{dim}_{\mathbb{Z}_{p \infty}} C \leq 1$, for all primes $p$.

Proof. Suppose that $\operatorname{dim}_{\mathbb{Z}_{p} \infty} C \geq 2$ were true for some prime $p$. Since $\operatorname{dim} C$ $\leq 2$ (if $\operatorname{dim} C \geq 3$, then $\operatorname{dim}(C \times D)>\operatorname{dim} C$ for every non-zero-dimensional compactum $D)$, it follows that

$$
\operatorname{dim}_{\mathbb{Z}_{(p)}} C=\operatorname{dim}_{\mathbb{Z}_{(p)}} C=\operatorname{dim}_{\mathbb{Z}_{p} \infty} C ;
$$

that is, $C$ is $p$-regular (cf. [6]), and hence

$$
\operatorname{dim}_{\mathbb{Z}_{(p)}}(C \times X)=\operatorname{dim}_{\mathbb{Z}_{(p)}} C+\operatorname{dim}_{\mathbb{Z}_{(p)}} X=2+2 .
$$

By assertion (iii) of Proposition 2.1 it follows that $\operatorname{dim}(C \times X)<4$, which yields a contradiction. This proves $\operatorname{dim}_{\mathbb{Z}_{p \infty}} C \leq 1$, for all primes $p$.

Thus, by Bockstein's theorem [11], $\operatorname{dim}_{\mathbb{Q} / \mathbb{Z}} C \leq 1$, and by the Countable Sum Theorem, $\operatorname{dim}_{\mathbb{Q} / \mathbb{Z}} B \leq 1$.

\section{ACKNOWLEDGMENTS}

This paper was written during the visit of the first and third authors to Ljubljana, in the summer of 1992 (and announced in [7]), on the basis of the agreement between the Slovenian Academy of Arts and Sciences and the Russian Academy of Sciences (1991-1995), and supported by a grant from the Ministry for Science and Technology of the Republic of Slovenia. 


\section{REFERENCES}

1. M. F. Bockstein, On homology invariants of topological spaces. I, Trudy Moskov. Mat. Obshch. 5 (1956), 3-80; English transl. in Amer. Math. Soc. Transl. Ser. 2, vol. 112, Amer. Math. Soc., Providence, RI, 1959.

2. V. G. Boltjanskii, An example of a two-dimensional compactum whose topological square has dimension equal to three, Dokl. Akad. Nauk SSSR 67 (1949), 597-599; English transl., Amer. Math. Soc. Transl., vol. 48, Amer. Math. Soc., Providence, RI, 1951, pp. 3-6.

3. A. N. Dranišnikov, Homological dimension theory, Uspekhi Mat. Nauk 43 (1988), 11-55; English transl., Russian Math. Surveys 43 (1988), 11-63.

4. __, On intersections of compacta in Euclidean space. II, Proc. Amer. Math. Soc. 113 (1991), 1149-1154.

5. A. N. Dranišnikov, D. Repovš, and E. V. Ščepin, On intersection of compacta of complementary dimension in euclidean space, Topology Appl. 38 (1991), 237-253.

6. __ On approximation and embedding problems for cohomological dimension, Topology Appl. (to appear).

7. _ On the failure of the Urysohn-Menger sum formula for cohomological dimension, Abstracts Amer. Math. Soc. 14 (1993), No. 93T-55-06.

8. J. Dydak, Cohomological dimension and metrizable spaces, Trans. Amer. Math. Soc. 337 (1993), 219-234.

9. J. Dydak and J. J. Walsh, Aspects of cohomological dimension for principle ideal domains, preliminary draft, Univ. of Tennessee, Knoxville, 1992.

10. K. Kuratowski, Topologie, Tome I, PWN, Warszawa, 1958.

11. V. I. Kuz'minov, Homological dimension theory, Uspekhi Mat. Nauk 23 (1968), 3-49; English transl., Russian Math. Surveys 23 (1968), 1-45.

12. M. Lavrent'ev, Contribution á la théorie des ensembles homéomorphes, Fund. Math. 6 (1924), 149.

13. L. R. Rubin, Characterizing cohomological dimension: The cohomological dimension of $A \cup B$, Topology Appl. 40 (1991), 233-263.

14. S. Spież, Imbeddings in $\mathbb{R}^{2 m}$ of $m$-dimensional compacta with $\operatorname{dim}(X \times Y)<2 m$, Fund. Math. 134 (1990), 105-115.

Institute for Mathematics, Physics, and Mechanics, University of Ljubljana, P. O. Box 64, Luubluana 61111 , Slovenia

Permanent address, A. N. Dranišnikov and E. V. Ščepin: Steklov Mathematical Institute, 42 Vavilova Street, 117966 Moscow GPS-1, Russia

E-mail address, D. Repovš: dusan.repovs@uni-lj.si

E-mail address, D. Repovš: repovs@itsictp̣.bitnet 